\title{
A Novel Technology for Measurements of Dielectric Properties of Extremely Small Volumes of Liquids
}

\author{
Wei-Na Liu ${ }^{1,2}$ \\ ${ }^{1}$ College of Physics and Electronic Engineering, Henan Normal University, Xinxiang 453007, China \\ ${ }^{2}$ Henan Key Discipline Open Laboratory of Electromagnetic Wave Detecting, Xinxiang 453007, China
}

Correspondence should be addressed to Wei-Na Liu; weinaliu926@163.com

Received 10 September 2015; Revised 22 November 2015; Accepted 29 November 2015

Academic Editor: Stefano Selleri

Copyright (C) 2016 Wei-Na Liu. This is an open access article distributed under the Creative Commons Attribution License, which permits unrestricted use, distribution, and reproduction in any medium, provided the original work is properly cited.

\begin{abstract}
A high sensitivity sensor for measurement radio frequency (RF) dielectric permittivity of liquids is described. Interference is used and parasitic effects are cancellation, which makes the sensor can catch weak signals caused by liquids with extremely small volumes. In addition, we present the relationship between transmission coefficient and permittivity of liquids under test (LUT). Using this sensor, quantitative measurements of the dielectric properties at $5.8 \mathrm{GHz}$ are demonstrated of LUTs. Experiments show that the proposed method only requires the volume of 160 nanoliters for liquids. Therefore, the technology can be used for RF spectroscopic analysis of biological samples and extremely precious liquids.
\end{abstract}

\section{Introduction}

RF dielectric properties of liquids of small volumes are of great interest for the development of biological and chemical fields [1-3]. Applications include dielectric studies of cells [4], medical diagnostic testing [5], and biological specimens [6] for lab-on-chip advancement. Dielectric spectroscopy that measures the dielectric properties has several advantages, such as being label-free, real-time monitoring of the samples, and being noninvasive [7]. However, application of RF dielectric properties of liquids at extremely low signal level is challenged, because low signal levels always submerge backgrounds that come from the measurement sensor [8]. One way to generate low signal is small volume sample, because large changes of the dielectric properties of samples with extremely small volumes can lead to small changes in the detected RF signals. Therefore, the sensitivity of permittivity measurement always decreases with the physical volume of the sample. At the same time, a novel technology for measurement of very small volumes of samples is particularly desirable for applications of biological and chemistry fields [9]. There have been many efforts and progress to improve measurement sensitivity and accuracy through different methods. These include transmission line [10], free space [11], and cavity techniques [12]. Nevertheless, parasitic effects [7-9] usually have a negative impact on the sensitivity of detection. The efforts are limited in counteracting parasitic effects and capturing very low signal that comes from extremely small volume [13] of the sample. So measurement of weak RF signal continues to be a technical challenge. At the same time, there also has been a difficulty in gaining dielectric properties of the liquids from the measured signals [14].

In the work, we propose a sensor for measurement of the dielectric properties of very small volumes of liquids at RF. Interference [7-9] is used to improve sensitivity of the sensor. So it can capture weak signals from extremely small volumes (nanoliter). In addition, the sensor can be used in contactless mode and possesses the quantitative extraction of the complex dielectric properties of the sample.

\section{Sensor Fabrication}

Figure 1(a) shows a schematic of the proposed structure. We can see that the sensor consists of two branches and two identical Wilkinson power dividers [15]. The two branches are composed of 180-degree reverse-phase and in-phase CPW(coplanar waveguide-) slot-line back-to-back balun [16]; one branch is filled with LUT; the other is filled with referenced liquid (RFL). An incoming microwave signal from port 1 is separated evenly into the two branches by the first power 


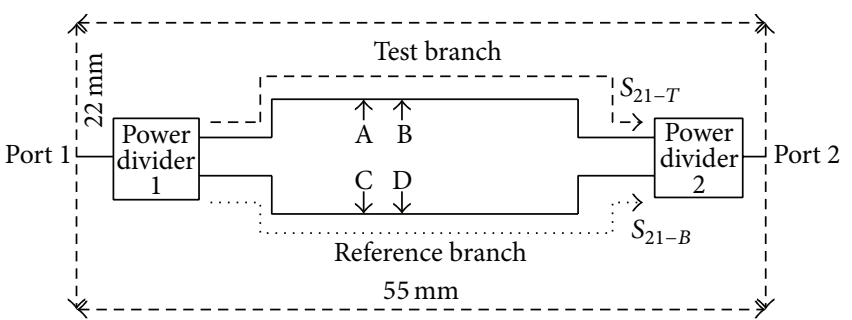

(a)

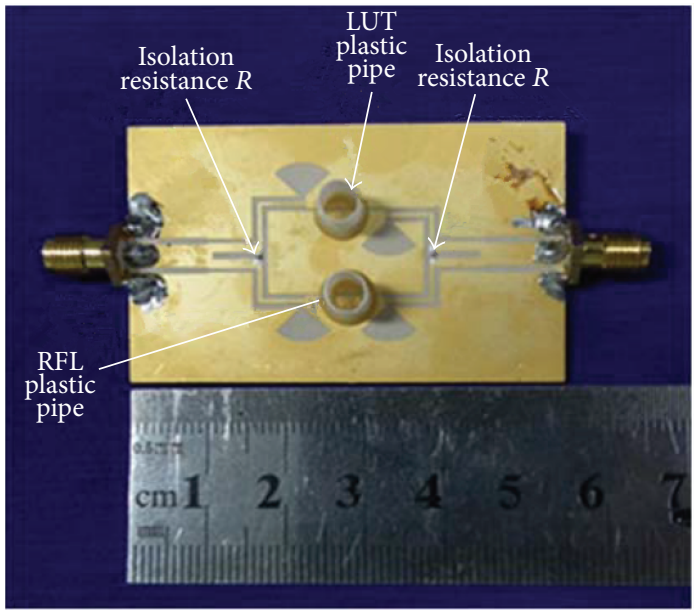

(b)

Figure 1: (a) Schematic of the proposed high sensitive sensor. (b) Fabricated sensor with plastic pipes.

divider; then signals will propagate to the two admittances and finally arrive to the second power divider [17].

Therefore, interference is used to improve measurement sensitivity greatly, which has been reported at radio frequency in [7-9]. If the two branches are filled with identical samples, the transmission $S_{21}$ is close to zero. Otherwise, small differences of the two dielectric properties will lead to large relative changes of $S_{21}$ between LUT and RFL. The sensor is designed by CPW (coplanar waveguide) and slot-line. A prototype is fabricated on an AD1000 substrate (relative permittivity is 10.2; substrate thickness is $1.6 \mathrm{~mm}$ ). The characteristic impedances of ports 1 and 2 are chosen as $50 \mathrm{ohm}$, for the convenience of process; the input/output characteristic impedance of the baluns is chosen as $75 \mathrm{ohm}$. Therefore, the even mode characteristic impedance of quarter wavelength section of the power divider and isolation resistance are 86.6 and $150 \mathrm{ohm}$, respectively. For the convenience of sample solution injection, LUT and RFL plastic pipes are attached on the sensor. The fabricated sensor with SMA connectors and plastic pipes is shown in Figure 1(b).

\section{Dielectric Properties Extraction Method}

Assuming that $S_{21-T}$ and $S_{21-B}$ are the signal transmission coefficients of the two branches, then the signal transmission coefficient from port 1 to port 2 can be expressed as

$$
S_{21}=S_{21-T}+S_{21-B} \text {. }
$$

If $T_{1}$ and $T_{2}$ express transmission coefficients of sections of $\mathrm{A}-\mathrm{B}$ and $\mathrm{C}-\mathrm{D}$, then

$$
S_{21}=S_{21-T r} \times T_{1}+S_{21-B r} \times T_{2}
$$

where $S_{21-T r}$ and $S_{21-B r}$ are the rest of signal path components except the A-B and C-D sections, respectively. For the convenience of test, two identical plastic pipes whose diameter and height equal $2 \mathrm{~mm}$ and $4 \mathrm{~mm}$ are glued at the A-B and C-D sections. And, for the REL cell,

$$
T_{2}=\frac{2 Z_{0}}{2 Z_{0}+Z_{2(\omega)}},
$$

where $Z_{0}$ is the characteristic impedance of transmission line and $Z_{2(\omega)}^{-1}=G_{2(\omega)}=j \omega C_{1}+j \omega C \varepsilon_{2} . C_{1}$ is the parasitic capacitance and $C$ is the capacitance of the liquid channels, which can change with variations of samples filled in the channel. If $\varepsilon_{2}$ and $\sigma_{2}$ are the complex permittivity and conductivity of the sample in the REL channel, respectively [18], then

$$
\varepsilon_{2}=\varepsilon_{2}^{\prime}+j\left(\varepsilon_{2}^{\prime \prime}-\frac{\sigma_{2}}{\varepsilon_{0} \omega}\right) .
$$

Similar relationship applies to LUT; the change of dielectric properties of it can change $C$ and $G$ with an amount of $\Delta C$ and $\Delta G$. Therefore, we have

$$
T_{1}=T_{2}+\Delta T .
$$

From formulas (2) and (5), we obtain

$$
S_{21}=S_{21-T r} \times\left(T_{2}+\Delta T\right)+S_{21-B r} \times T_{2} .
$$

Furthermore, $S_{21-T r} \times T_{2}+S_{21-B r} \times T_{2}=0$. For our channels, $T_{1,2} \ll 1$, so

$$
\begin{aligned}
S_{21} & =S_{21-T r} \times \Delta T \\
& =S_{21-T r} \times 2 Z_{0} \frac{Z_{2(\omega)}-Z_{1(\omega)}}{\left[2 Z_{0}+Z_{1(\omega)}\right]\left[2 Z_{0}+Z_{2(\omega)}\right]} \\
& \approx 2 Z_{0} j \omega S_{21-T r}\left(\varepsilon_{1}-\varepsilon_{2}\right) C,
\end{aligned}
$$

where $\varepsilon_{1}$ and $\varepsilon_{2}$ are permittivity of LUT and RFL, respectively. Expression (7) shows that the difference of dielectric properties is direct relationship to the measured $S_{21}$ of the sensor, 
TABLE 1: Measurement results for methanol, M-E, M-P, and n-propanol.

\begin{tabular}{lcccccc}
\hline LUT & $\varepsilon^{\prime}$ & Reference [19] & Relative error (\%) & $\varepsilon^{\prime \prime}$ & Reference [19] & Relative error (\%) \\
\hline Methanol & 31.013 & 30.279 & 2.42 & -8.126 & -7.904 & -2.81 \\
M-E & 22.287 & 21.734 & 2.54 & -10.025 & -9.818 & -2.11 \\
M-P & 17.556 & 17.915 & -2.01 & -10.356 & -10.212 & -1.41 \\
Ethanol & 13.986 & 14.403 & -2.89 & -9.389 & -9.524 & 1.42 \\
n-Propanol & 8.126 & 7.910 & 2.73 & -7.669 & -7.831 & 2.07 \\
\hline
\end{tabular}

where $C$ is lossless in expression (7), since the tubes and the substrate (Rogers TMM10) have very low dielectric loss in design frequency $(5.8 \mathrm{GHz})$.

If considering nonidealities of the two power dividers and small differences in the size of the two channels, the transmission coefficient $S_{21}$ can be written as follows:

$$
\begin{aligned}
S_{21} & =2 Z_{0} j \omega\left[S_{21-T r}\left(\varepsilon_{1}-\varepsilon_{2}\right) C\right]+S_{21-T r}\left[\varepsilon_{1} \Delta C\right. \\
& \left.+\Delta C_{1}\right]+\left(S_{21-T r} T_{2}+S_{21-B r} T_{1}\right)\left(C \varepsilon_{1}+C_{1}\right) \\
& =\left[S_{21-T r} \Delta C+\left(S_{21-T r} T_{2}+S_{21-B r}\right) C\right. \\
& \left.+2 Z_{0} j \omega S_{21-T r} C\right] \varepsilon_{1}+S_{21-T r} \Delta C_{1}+\left[S_{21-T r} T_{2}\right. \\
& \left.+S_{21-B r} T_{1}\right] C_{1}-2 Z_{0} j \omega S_{21-T r} C \varepsilon_{2}=M \varepsilon_{1}+N \varepsilon_{2} \\
& +P,
\end{aligned}
$$

where $C_{1}$ is parasitic capacitance and lossless in (7) and $\Delta C, \Delta C_{1}$ are changes of channel and parasitic capacitances, respectively. In the work, deionized water is chosen as RFL. So coefficients of $M, N$, and $P$ can be determined from the tested $S_{21}$ of two liquids with known permittivity.

\section{Results and Discussions}

On the basis of the proposed method, transmission parameters of $S_{21}$ are tested with a network analyzer. During experiments, deionized water is the RFL, and methanol, ethanol-propanol, M-E, and M-P are chosen as tested liquids. M-E (M-P) is mixtures of methanol and ethanol (methanol and n-propanol) with identical mole fraction. Measurements were performed at room temperature of $25^{\circ} \mathrm{C}$. The magnitude of $S_{21}$ is shown in Figure 2. The measurement results demonstrate convincingly the theoretical analysis noted above. That is, the transmission coefficients of the sensor have inevitable connection with the permittivity difference between the RFL and LUT.

The transmission of $S_{21}$ is about $-70 \mathrm{~dB}$, when the two channels are placed with the same materials (deionized water). It also exhibits a strong dependence on the dielectric properties of LUT.

We chose deionized water $(\sim 80)$ and acetone $(\sim 20.5)$ as calibration liquids and permittivity of LUT is computed from the measured $S_{21}$, accordingly from (8). The results are shown in Table 1. We can see that the measured results are in good agreement with reference [19].

One way to illustrate the sensitivity of the proposed method is shown in Figure 3, which compare the amplitude

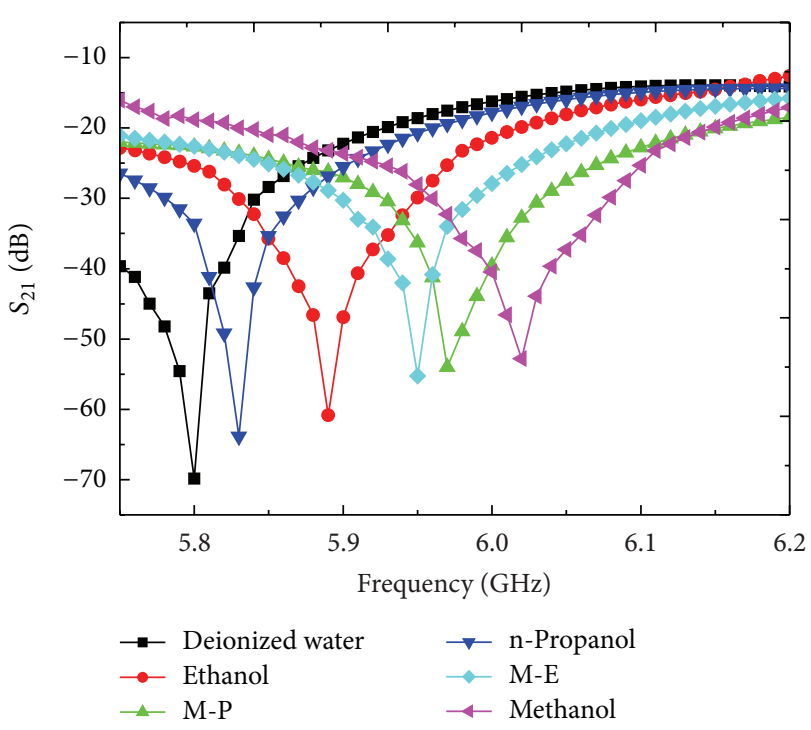

FIGURE 2: Measured $S_{21}$ for different LUT under the RFL which is deionized water.

of $S_{21}$ for different LUTs. In the process of this experiment, the A-B section which is reference channel is filled full in deionized water, and the LUT channel residing in the C-D section is filled in ethanol with different molar concentration, respectively. From Figure 3, the maximum $S_{21}$ changes are $\sim 0.22 \mathrm{~dB}$ for shielded coplanar waveguide (S-CPW) [14] method. However, the proposed method exhibits large $S_{21}$ changes for the same permittivity difference between LUT and RFL, which indicates high sensitivity. The dielectric constant values obtained in [19] are used.

Another way of measurement sensitivity is defined as the relative change of interference frequency along with volume of different LUTs. Figure 4 shows the test results of sensitivity on the basis of dielectric properties of LUTs calculated above. Five sampling valves of permittivity correspond to five LUTs; they are methanol, M-E, M-P, ethanol, and n-propanol. It can be seen that the larger the volume of LUTs, the higher the sensitivity when the LUTs remain the same. So the sensitivity of the measurement increases with volumes of LUTs. However, the volumes of the channel will not affect the sensitivity of the sensor if volume is greater than or equal to $160 \mathrm{~nL}$ as can be seen from Figure 4, because two curves ( $V=140$ nanoliters and $V=160$ nanoliters) have already overlapped. That is, the volume of the LUT only requires $160 \mathrm{n} \mathrm{L}$. Compared with [13], the proposed method is much more sensitive. At the same time, the detector requires 


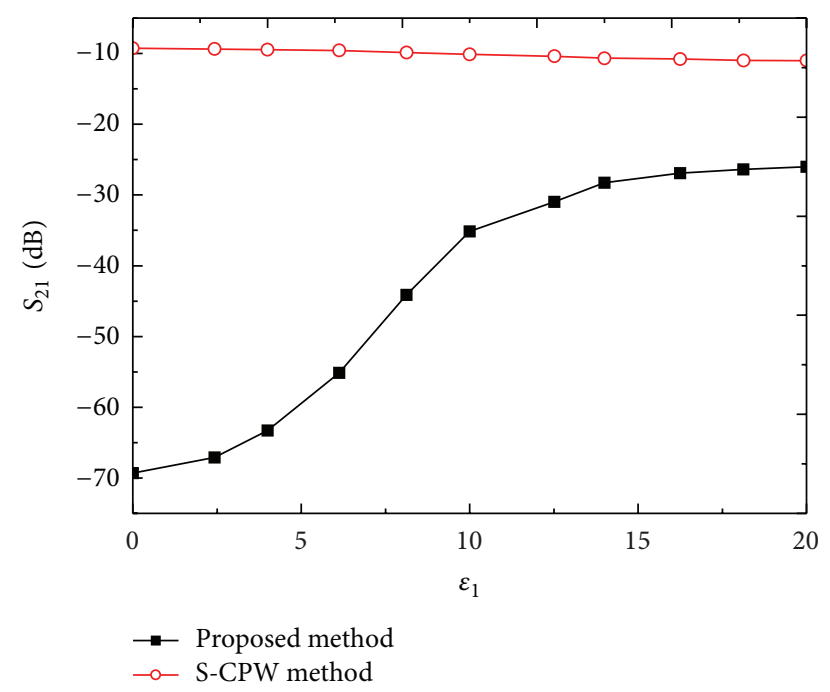

FIGURE 3: The scattering parameters $S_{21}$ change with various differences of permittivity for two methods.

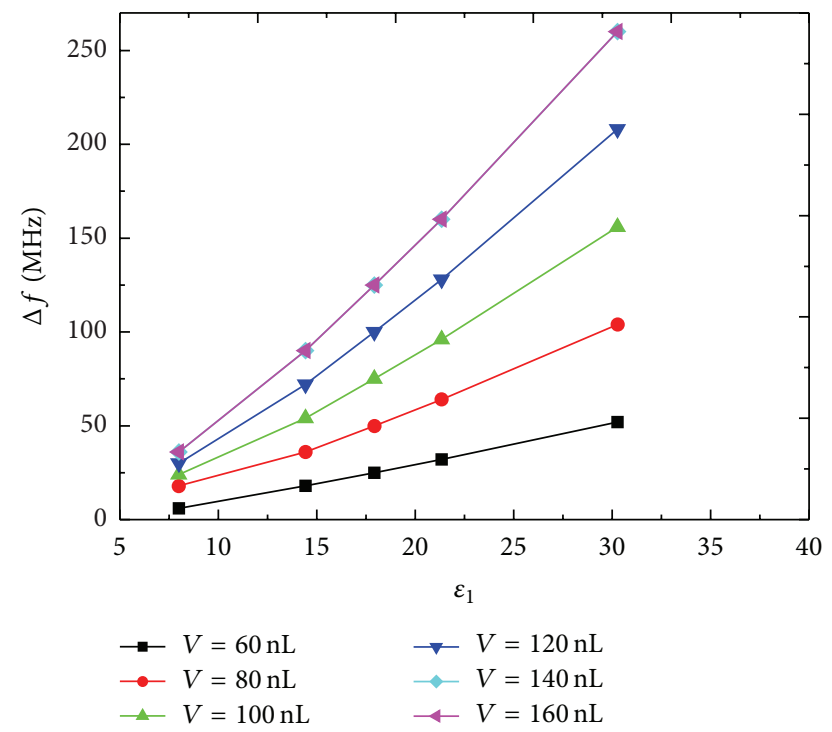

FIGURE 4: Variation of cancellation frequency with permittivity of LUT calculated by the proposed method.

the smallest volume of 160 nanoliters. It is also shown that the proposed method is very high sensitive for detecting signal generated by liquids with extremely small volumes. In previous work, an extremely small sample ( 200 nanoliters) has been realized in [20]. Compared with it, a smaller sample volume has been required by using the proposed method.

\section{Conclusion}

In summary, a high sensitive sensor for measurement permittivity with very small volumes of liquids is demonstrated. There are two attractive features of the proposed method: the measurement is very high sensitive and permittivity of liquids can be carried out with small volume. Therefore, the sensor can be used for measurement dielectric spectrum for biological samples or extremely precious liquids. At the same time, we analyze deeply the relationship between transmission coefficient and dielectric properties of LUT. There are some issues that need to be studied, such as bandwidth and miniaturization.

\section{Conflict of Interests}

The author declares that there is no conflict of interests regarding the publication of this paper.

\section{Acknowledgment}

This project is supported by the Doctor Start-Up fund of $\mathrm{He}$ Nan Normal University (no. 5101029170255).

\section{References}

[1] F. Bourdiol, D. Dubuc, K. Grenier, F. Mouchet, L. Gauthier, and E. Flahaut, "Quantitative detection of carbon nanotubes in biological samples by an original method based on microwave permittivity measurements," Carbon, vol. 81, no. 1, pp. 535-545, 2015.

[2] R. J. Sengwa, S. Choudhary, and V. Khatri, "Microwave dielectric spectra and molecular relaxation in formamide-N,Ndimethylformamide binary mixtures," Spectrochimica Acta Part A: Molecular and Biomolecular Spectroscopy, vol. 82, no. 1, pp. 279-282, 2011.

[3] K. Kim, N. Kim, S.-H. Hwang, Y.-K. Kim, and Y. Kwon, "A miniaturized broadband multi-state reflectometer integrated on a silicon MEMS probe for complex permittivity measurement of biological material," IEEE Transactions on Microwave Theory and Techniques, vol. 61, no. 5, pp. 2205-2214, 2013.

[4] K. Asami, "Dielectric properties of microvillous cells simulated by the three-dimensional finite-element method," Bioelectrochemistry, vol. 81, no. 1, pp. 28-33, 2011.

[5] P.-O. Bagnaninchi, M. Dikeakos, T. Veres, and M. Tabrizian, "Complex permittivity measurement as a new noninvasive tool for monitoring in vitro tissue engineering and cell signature through the detection of cell proliferation, differentiation, and pretissue formation," IEEE Transactions on Nanobioscience, vol. 3, no. 4, pp. 243-250, 2004.

[6] Z. Ji, S. C. Hagness, J. H. Booske, S. Mathur, and M. L. Meltz, "FDTD analysis of a gigahertz TEM cell for ultrawideband pulse exposure studies of biological specimens," IEEE Transactions on Biomedical Engineering, vol. 53, no. 5, pp. 780$789,2006$.

[7] C. Song and P. Wang, "A radio frequency device for measurement of minute dielectric property changes in microfluidic channels," Applied Physics Letters, vol. 94, no. 2, Article ID 023901, 2009.

[8] C. Song and P. Wang, "On-chip cancellation of parasitic effects for dielectric permittivity measurement," in Proceedings of the IEEE MTT-S International Microwave Symposium Digest, pp. 131-134, Atlanta, Ga, USA, June 2008.

[9] Y. Yang, H. Zhang, J. Zhu et al., "Distinguishing the viability of a single yeast cell with an ultra-sensitive radio frequency sensor," Lab on a Chip-Miniaturisation for Chemistry and Biology, vol. 10 , no. 5, pp. 553-555, 2010. 
[10] D. Beruhe, F. M. Ghannouchi, and P. Savard, "A comparative study of four open-ended coaxial probe models for permittivity measurements of lossy dielectric/biological materials at microwave frequencies," IEEE Transactions on Microwave Theory and Techniques, vol. 44, no. 10, pp. 1928-1934, 1996.

[11] T. Tosaka, K. Fujii, K. Fukunaga, and A. Kasamatsu, "Development of complex relative permittivity measurement system based on free-space in 220-330-GHz range," IEEE Transactions on Terahertz Science and Technology, vol. 5, no. 1, pp. 102-109, 2015.

[12] M. Tuhkala, J. Juuti, and H. Jantunen, "Determination of complex permittivity of surfactant treated powders using an open-ended coaxial cavity resonator," Powder Technology, vol. 256, pp. 140-145, 2014.

[13] W. Daseler, H.-J. Steinhoff, and A. Redhardt, "A new method for the determination of the permittivity of small samples in the microwave range and its application to hemoglobin single crystals," Journal of Biochemical and Biophysical Methods, vol. 22, no. 1, pp. 69-82, 1991.

[14] A.-L. Franc, E. Pistono, and P. Ferrari, "Characterization of thin dielectric films up to $\mathrm{mm}$-wave frequencies using patterned shielded coplanar waveguides," IEEE Microwave \& Wireless Components Letters, vol. 22, no. 2, pp. 100-102, 2012.

[15] X. Wang, K.-L. Wu, and W.-Y. Yin, "A compact gysel power divider with unequal power-dividing ratio using one resistor," IEEE Transactions on Microwave Theory and Techniques, vol. 62, no. 7, pp. 1480-1486, 2014.

[16] C.-H. Ho, L. Fan, and K. Chang, "New uniplanar coplanar waveguide hybrid-ring couplers and magic-T's," IEEE Transactions on Microwave Theory and Techniques, vol. 42, no. 12, pp. 2440-2448, 1994.

[17] W.-N. Liu, Y. Yang, and K.-M. Huang, "A radio frequency sensor for measurement of small dielectric property changes," Journal of Electromagnetic Waves and Applications, vol. 26, no. 8-9, pp. 1180-1191, 2012.

[18] D. M. Pozar, Microwave Engineering, John Wiley \& Sons, 2009.

[19] S. Mashimo, T. Umehara, and H. Redlin, "Structures of water and primary alcohol studied by microwave dielectric analyses," The Journal of Chemical Physics, vol. 95, no. 9, pp. 6257-6260, 1991.

[20] A. Kozhevnikov, "Wideband radio-frequency device for measurements of dielectric properties of small volumes of liquids," Measurement Science \& Technology, vol. 21, no. 4, pp. 89-94, 2010. 


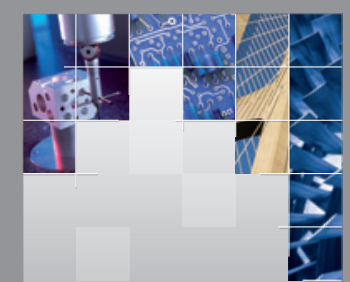

\section{Enfincering}
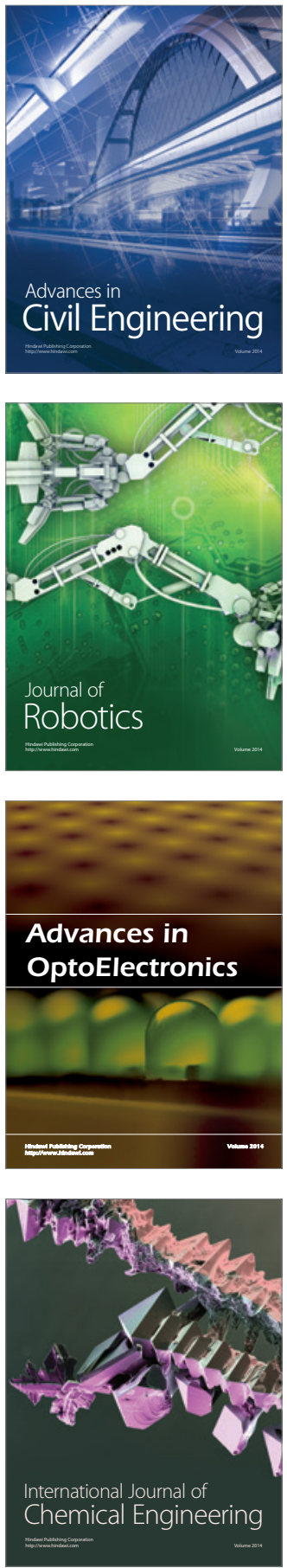

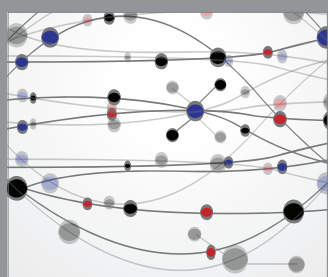

The Scientific World Journal

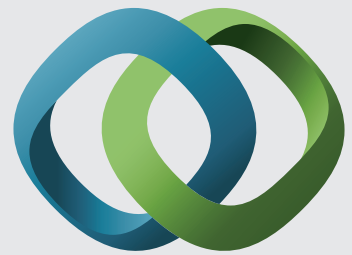

\section{Hindawi}

Submit your manuscripts at

http://www.hindawi.com
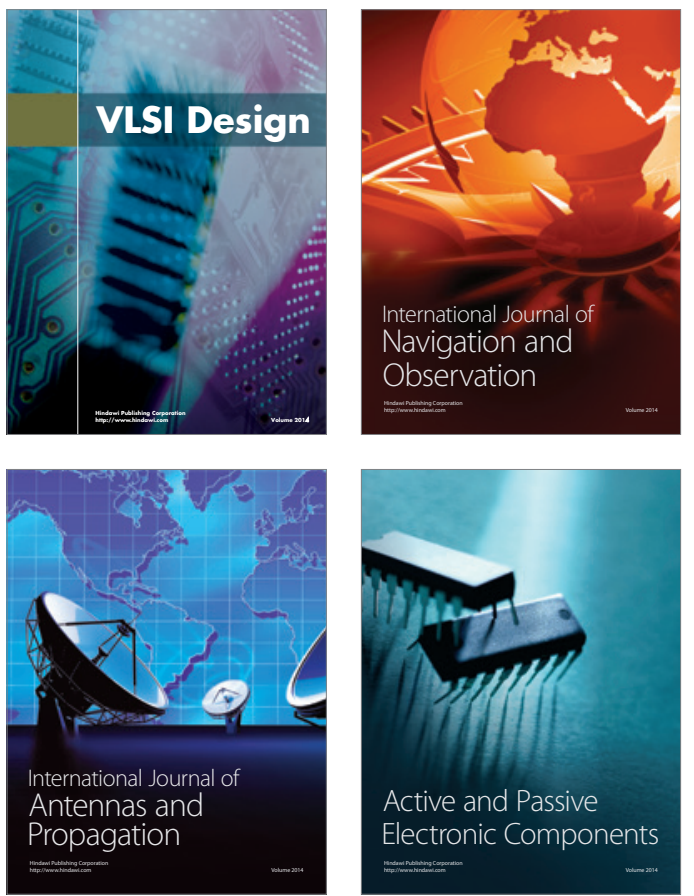
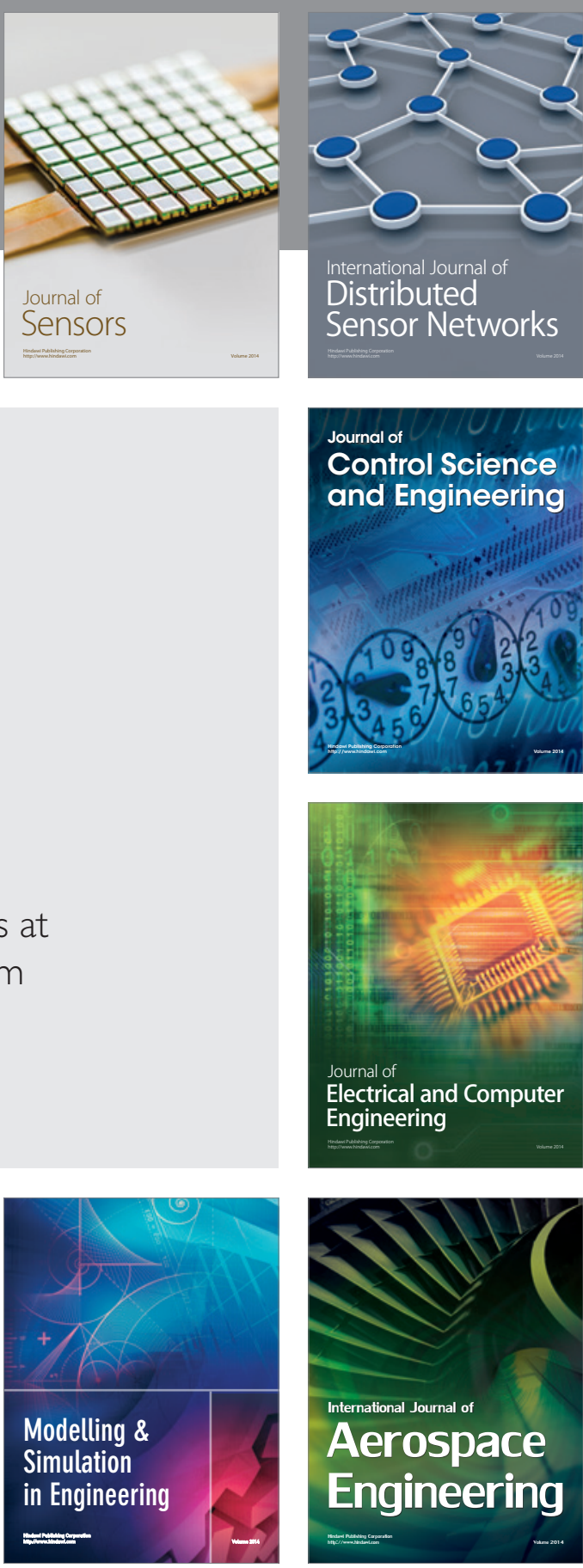

International Journal of

Distributed

Sensor Networks

Journal of

Control Science

and Engineering
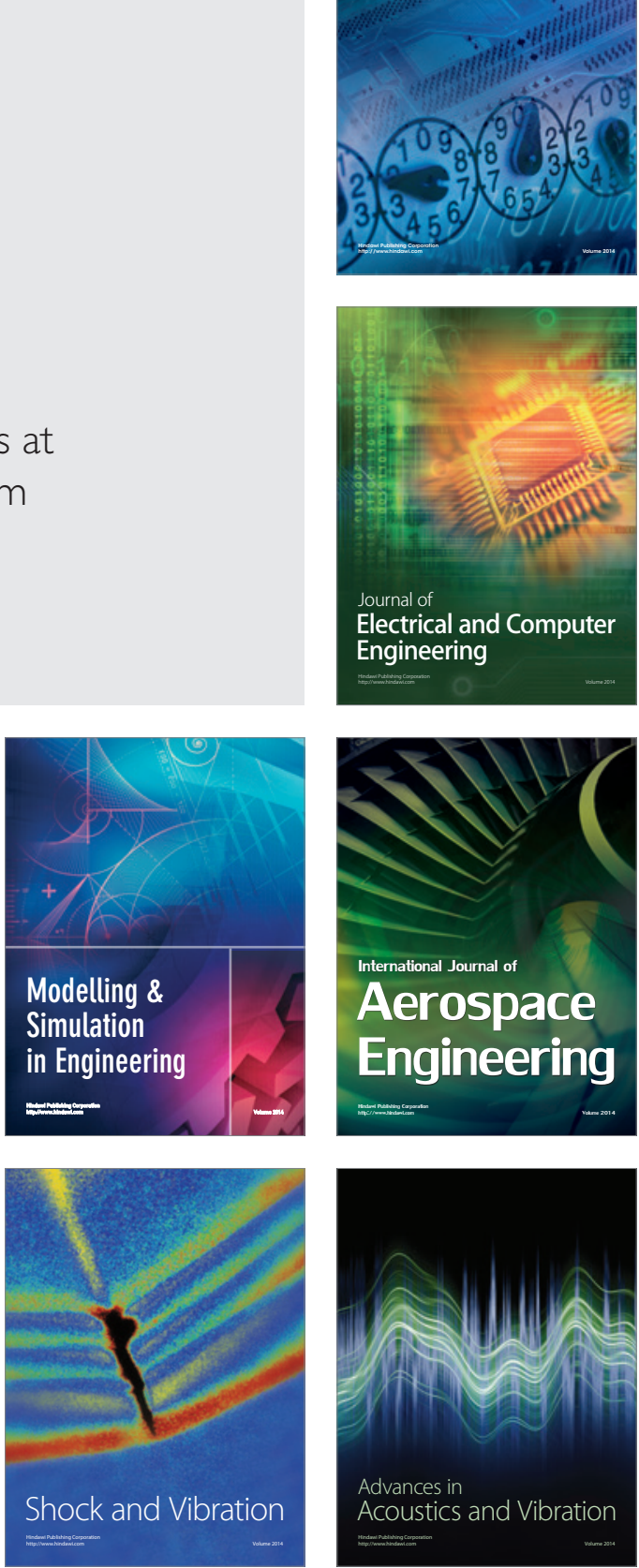